\title{
MicroRNAs and pharmacogenomics
}

Pharmacogenomics studies the influence of genomics on drug response safety and efficacy. Although research in this field was initiated many years ago, few functional applications are currently in use at the clinic. MicroRNAs (miRNAs) are short noncoding RNAs that bind genes and silence their expression. MiRNAs are encoded by the genome and expressed in all animal cells. MiRNAs are predicted to target approximately half of all human genes, and as a result regulate many cellular processes. The current focus of pharmacogenomics is the identification of polymorphisms in candidate genes coding for drug-metabolizing enzymes, drug transporters and drug targets. Here we call for the evaluation of miRNAs as an additional regulatory layer affecting pharmacogenomics. To illustrate the potential of miRNAs for affecting drug response we performed in silico evaluation of miRNA binding regions in genes known to affect drug response. We suggest that miR-133 and miR-137 may affect VKORC1 expression while miR-22 may affect MTHFR expression. We propose that miRNAs play a central role as a novel regulatory layer affecting drug metabolism and drug targets, and thus should be taken into consideration when conducting pharmacogenomic studies.

\section{KEYWORDS: gene expression microRNA miRNA pharmacogenetics pharmacogenomics regulation}

\section{Pharmacogenomics: past, present \& future}

Pharmacogenomics studies the contribution of genomes, transcriptomes and proteomes in determining drug-response phenotypes (safety and efficacy). The major goal of pharmacogenomics research is the development of genotype- or transcriptome-based predictive tests of drug efficacy or toxicity. Although research in this field began more than 50 years ago [1], few functional applications are currently implemented in the clinical setting [2]. An evolving novel world of small regulatory RNAs, mainly governed by so-called 'microRNAs' (miRNAs), which are coded in our genomes and are implicated in post-transcriptional control, offers an additional layer of intricate regulation of drug response by controlling the expression of drugmetabolizing enzymes, drug transporters and drug targets. However, a prerequisite for understanding the role of miRNAs in drug response phenotypes is the reliable understanding of the interaction between the miRNA molecules and their myriad gene targets.
MiRNAs: new players, new concepts

MiRNAs represent a relatively new discipline in biomedical research. Identified in 1993 [3], and widely appreciated by 2001 [4-6], miRNAs are short ( 22 nucleotide) noncoding RNAs expressed in all animal and plant cells. These small RNA molecules bind the $3^{\prime}$ ends of expressed mRNA transcripts and mark them for degradation and/or translation inhibition (Figure 1A) [7]. There are hundreds of different miRNAs in animals, some of which are expressed at thousands of copies per cell. Each miRNA is predicted to target and bind, via a short six-nucleotide region in the 3'-UTR (FIgure 1B \& C), approximately 200 genes, leading to an estimated regulation, in humans, of approximately half of the transcribed mRNAs (or transcriptome; [8]). As a result, and not surprisingly, many cellular and physiological processes in health and disease were associated with changes in miRNA expression. Among these are differentiation, development, growth, apoptosis, cancer and neurological disorders (for example see $[9,10])$.

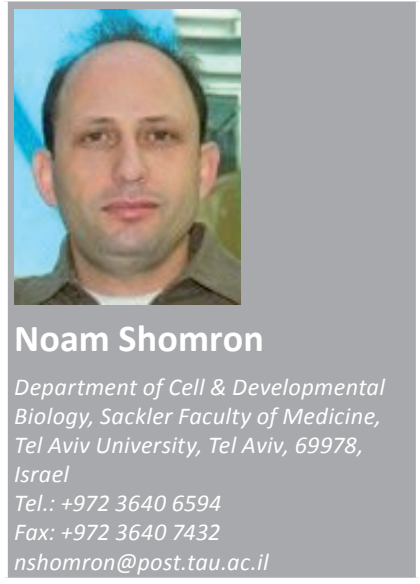

future medicine $^{\text {profs }}$ fs 


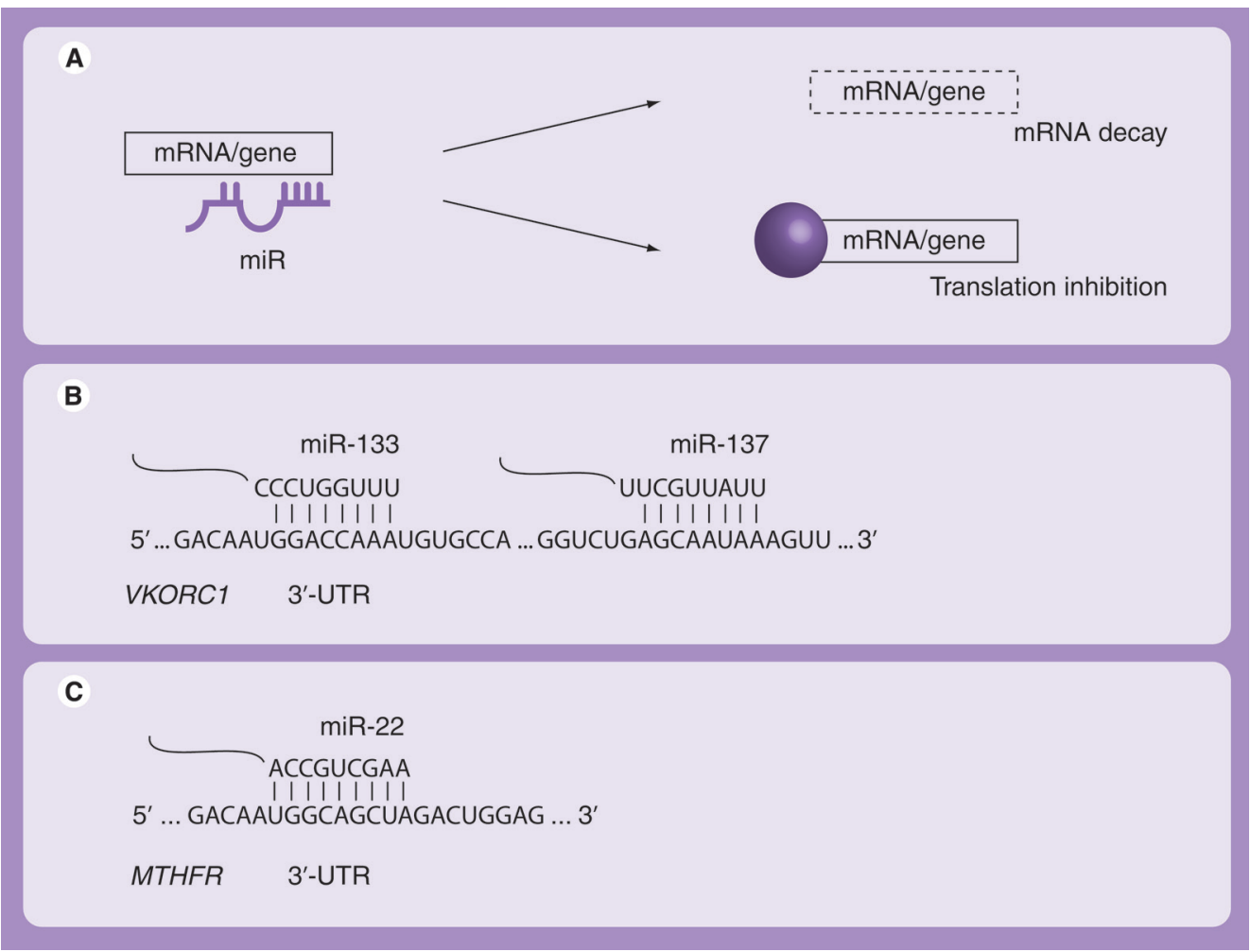

Figure 1. Regulation of gene expression by microRNAs. (A) An interaction between a miR and a protein-coding gene. A binding region that is a minimum of six nucleotides between the $5^{\prime}$ end of the miR and the $3^{\prime}$-UTR of the target gene is required to confer inhibition through miR degradation or translation inhibition. The interaction between miR-133 and miR-137 is shown on the 3'-UTR of VKORC1 (B) and between miR-22 and the MTHFR 3'-UTR (C). We note that all three miR-binding sites are highly conserved in evolution, indicating their genuine functionality. miR: MicroRNA.

The current focus of pharmacogenomics is primarily on studying the phenotypic effects of SNPs and other genetic variations (such as copy number variations) in candidate drugmetabolizing and drug-target genes. These concerted efforts have so far led to very limited recommendations for clinical use by the US FDA [11].

Recently, researchers have called for an unbiased genome-wide comprehensive analysis in order to map the entire correlation to drug efficacy [12]. This notion coincides with high-throughput sequencing technologies (also known as 'next-generation' or 'deep-sequencing' technologies) becoming available for massive nucleotide sequencing, which allow the sequencing of entire genomic regions and not only SNPs [13]. These new technologies can also be valuable for studying how epigenetics affects drug response through, for example, DNA-methylation patterns [14].

Here we call for the incorporation of miRNAs as an additional pharmacogenomics research focus. If our basic assumption is that the expression levels of genes coding for drug-metabolizing enzymes, drug transporters, or drug targets modify drug efficacy or toxicity, basing pharmacogenomics studies on DNA-sequence analysis alone would strongly bias experimental interpretation. We suggest that many of the intracellular levels of the candidate genes are also regulated by miRNAs.

There are several ways in which miRNAs can control the expression of genes whose products affect drug response. Given the presence of a miRNA binding site in a gene's 3'-UTR, at least one additional prerequisite is necessary: a change in the cellular miRNA levels; SNPs or other inherited modifications in the miRNA binding site on the 3'-UTR (a miRNA binding site can also be generated de novo); sequence modification in the miRNA gene itself affecting its expression, biogenesis, stability or its binding affinities to the target gene.

\section{Some established \& tentative examples}

These types of interactions were observed by a few research teams in the context of pharmacogenomics-relevant genes. The drug-metabolizing 
enzyme CYP1B1, for example, was shown to be highly expressed in various types of malignant cancers and was also shown to be actively regulated by miR-27b [15]. Another illustration is the polymorphism in a miRNA binding site on the dihydrofolate reductase (DHFR) gene, which leads to loss of miRNA binding in its 3'-UTR and consequently to its overexpression and to methotrexate resistance [16].

However, these examples are two of the few published so far regarding miRNA effects on drug-response phenotypes. Here, we propose other intriguing examples, currently tentative, that need to be explored. The use of the anticoagulant warfarin may be made safer and more effective by considering an individual's genotype for cytochrome P450, family 2, subfamily C, polypeptide 9 (CYP2C9) and vitamin $\mathrm{K}$ epoxide reductase (VKORC1) [17]. By using available bioinformatics tools [18] we have identified two highly evolutionary conserved extended binding sites for miR-133 and miR-137 on VKORC1, suggesting an effective and strong regulation by these miRNAs.

Thus, the efficacy of warfarin could be altered in the event that at least one of these miRNAs is modified in an individual, leading to aberrant regulation of VKORC1 expression. Of note, miR-137 was reported to be associated with hematopoiesis [19].

Another example we offer is based on the 5,10-methylenetetrahydrofolate reductase $(M T H F R)$ gene, which is a key enzyme for intracellular folate homeostasis and metabolism. Two common MTHFR polymorphisms, leading to different enzyme activities, have been associated with susceptibility to cancer [20]. The gene's 3'-UTR has solid predictions for extremely conserved miRNA binding sites, one of which - miR-22 - was identified as being altered in cancer cells [21]. Thus, the anecdotal miRNA-target interactions brought forward here exemplify the need to assess the levels of drug-metabolizing genes in the cellular context when administrating drugs.

A study evaluating stress response on individual lymphoblastoid cells indicated that particular gene signatures can serve as predictors for cellular response in a variable population of individuals [22]. Since miRNAs were found to classify human disease with better precision than gene expression [23], we predict that they will also categorize drug response in a superior manner. Therefore, we call for a comprehensive study to decipher the roles that miRNAs play in pharmacogenomics.
For each drug-response experiment, we propose to conduct high-throughput miRNA profiling, prediction of miRNA binding sites and sequencing of all the relevant $3^{\prime}$-UTRs (using technologies such as those described in [13]). While the latter is most time and budget consuming, the matches between the three layers of information will result in an unbiased identification of miRNA-associated pharmacogenomic principles.

Studying the genetic basis of drug response could help clarify mechanisms of drug action and facilitate development of genotype-based, transcriptome-based or proteome-based predictive tests of drug efficacy and toxicity. The regulatory effect of miRNAs on gene levels is not always dominant or intense, yet they exhibit an ability to shift global gene expression in an appreciable manner [24,25]. We observed interindividual stochastic expression of miRNAs in normal human tissues [Sномron ET AL., UNPUBLished DATA] suggesting that the response to drugs might be hardwired in the levels of these cellular regulators. We suggest that our current knowledge of miRNAs involved in pharmacogenomics is only the tip of the iceberg, and that many of these variables will confer direct regulation on drug-metabolizing enzymes, drug transporters, drug targets or up-/down-stream drug-target proteins involved in drug response. MiRNA expression and regulatory mapping will enable the development of better pharmacogenomic tools, and could also be instrumental in new drug-development projects.

We have discussed here high expectations for the miRNA world to present a new regulatory layer affecting drug metabolism. We are confident that, when following basic efficacy and toxicity studies, in combination with miRNA analysis and 3'-UTR profiling, miRNAs will live up to their potential.

Financial \& competing interests disclosure

The research at the Shomron laboratory is supported by grants from the Chief Scientist Office, Ministry of Health, Israel (3-4876), the Kurz-Lion Foundation, the Ori Levi Foundation for Mitochondrial research, Tel Aviv University, Sackler Faculty of Medicine, Schreiber Fellowship and the Wolfson Charitable Fund. The author has no other relevant affliations or financial involvement with any organization or entity with a financial interest in or financial conflict with the subject matter or materials discussed in the manuscript apart from those disclosed.

No writing assistance was utilized in the production of this manuscript. 


\section{Bibliography}

1 Gurwitz D, Motulsky AG: Drug reactions, enzymes, and biochemical genetics: 50 years later. Pharmacogenomics 8(11), 1479-1484 (2007).

2 Holmes MV, Shah T, Vickery C, Smeeth L, Hingorani AD, Casas JP: Fulfilling the promise of personalized medicine? Systematic review and field synopsis of pharmacogenetic studies. PLoS One 4(12), E7960 (2009).

3 Lee RC, Feinbaum RL, Ambros V: The C. elegans heterochronic gene lin- 4 encodes small RNAs with antisense complementarity to lin-14. Cell 75(5), 843-854 (1993).

4 Lee RC, Ambros V: An extensive class of small RNAs in Caenorhabditis elegans. Science 294(5543), 862-864 (2001).

5 Lau NC, Lim LP, Weinstein EG, Bartel DP: An abundant class of tiny RNAs with probable regulatory roles in Caenorhabditis elegans. Science 294(5543), 858-862 (2001).

6 Lagos-Quintana M, Rauhut R, Lendeckel W, Tuschl T: Identification of novel genes coding for small expressed RNAs. Science 294(5543), 853-858 (2001).

7 Chekulaeva M, Filipowicz W: Mechanisms of miRNA-mediated post-transcriptional regulation in animal cells. Curr. Opin. Cell Biol. 21(3), 452-460 (2009).

8 Shomron N, Golan D, Hornstein E: An evolutionary perspective of animal microRNAs and their targets. J. Biomed. Biotechnol. 594738 (2009).

9 Hornstein E, Shomron N: Canalization of development by microRNAs. Nat Genet. 38(Suppl.) S20-S24 (2006).
10 Iorio MV, Croce CM: MicroRNAs in cancer: small molecules with a huge impact. J. Clin. Oncol. 27(34), 5848-5856 (2009).

11 Pendergast MK: Regulatory agency consideration of pharmacogenomics. Exp. Biol. Med. (Maywood) 233(12), 1498-1503 (2008).

12 Gurwitz D, McLeod HL: Genome-wide association studies: powerful tools for improving drug safety and efficacy. Pharmacogenomics 10(2), 157-159 (2009).

13 Metzker ML: Sequencing technologies - the next generation. Nat Rev Genet. 11(1), 31-46 (2010).

14 Hodges E, Smith AD, Kendall J et al.: High definition profiling of mammalian DNA methylation by array capture and single molecule bisulfite sequencing. Genome Res. 19(9), 1593-1605 (2009).

15 Tsuchiya Y, Nakajima M, Takagi S, Taniya T, Yokoi T: MicroRNA regulates the expression of human cytochrome P450 1B1. Cancer Res. 66(18), 9090-9098 (2006).

16 Mishra PJ, Humeniuk R, Mishra PJ, Longo-Sorbello GS, Banerjee D, Bertino JR: A miR-24 microRNA binding-site polymorphism in dihydrofolate reductase gene leads to methotrexate resistance. Proc. Natl Acad. Sci. USA 104(33), 13513-13518 (2007).

17 Wadelius M, Pirmohamed M: Pharmacogenetics of warfarin: current status and future challenges. Pharmacogenomics $J$. 7(2), 99-111 (2007).

18 Rajewsky N: MicroRNA target predictions in animals. Nat Genet. 38(Suppl.) S8-S13 (2006).
19 Choong ML, Yang HH, McNiece I: MicroRNA expression profiling during human cord blood-derived CD34 cell erythropoiesis. Exp. Hematol. 35(4), 551-564 (2007).

20 De Mattia E, Toffoli G: C677T and A1298C MTHFR polymorphisms, a challenge for antifolate and fluoropyrimidine-based therapy personalisation. Eur. J. Cancer 45(8), 1333-1351 (2009).

21 Kawahigashi Y, Mishima T, Mizuguchi Y et al.: MicroRNA profiling of human intrahepatic cholangiocarcinoma cell lines reveals biliary epithelial cell-specific microRNAs. J. Nippon Med. Sch. 76(4), 188-197 (2009).

22 Fry RC, Svensson JP, Valiathan C et al.: Genomic predictors of interindividual differences in response to DNA damaging agents. Genes Dev. 22(19), 2621-2626 (2008).

23 Lu J, Getz G, Miska EA et al.: MicroRNA expression profiles classify human cancers. Nature 435(7043), 834-838 (2005).

24 Lim LP, Lau NC, Garrett-Engele P et al.: Microarray analysis shows that some microRNAs downregulate large numbers of target mRNAs. Nature 433(7027), 769-773 (2005).

25 Nielsen CB, Shomron N, Sandberg R, Hornstein E, Kitzman J, Burge CB. Determinants of targeting by endogenous and exogenous microRNAs and siRNAs. RNA 13(11), 1894-1910 (2007). 